\title{
BMJ Open Gender inequalities in the association between area deprivation and perceived social support: a cross-sectional multilevel analysis at the municipality level in Germany
}

\author{
Emily Mena, ${ }^{1,2}$ Lars Eric Kroll, ${ }^{3}$ Werner Maier, ${ }^{4}$ Gabriele Bolte ${ }^{1,2}$
}

To cite: Mena E, Kroll LE, Maier W, et al. Gender inequalities in the association between area deprivation and perceived social support: a cross-sectional multilevel analysis at the municipality level in Germany. BMJ Open 2018;8:e019973. doi:10.1136/ bmjopen-2017-019973

- Prepublication history for this paper is available online. To view these files, please visit the journal online (http://dx.doi org/10.1136/bmjopen-2017019973).

Received 5 0ctober 2017 Revised 20 December 2017 Accepted 9 February 2018

Check for updates

${ }^{1}$ Department of Social Epidemiology, Institute of Public Health and Nursing Research, University of Bremen, Bremen, Germany

${ }^{2}$ Health Sciences Bremen, University of Bremen, Bremen, Germany

${ }^{3}$ Department of Epidemiology and Health Monitoring, Robert Koch Institute, Berlin, Germany ${ }^{4}$ Institute of Health Economics and Health Care Management, Helmholtz Zentrum München, German Research Center for Environmental Health (GmbH), Neuherberg, Germany

Correspondence to

Emily Mena;

e.mena@uni-bremen.de

\section{ABSTRACT}

Objectives To investigate the association between area deprivation at municipality level with low perceived social support, independent of individual socioeconomic position and demographic characteristics. To assess whether there are gender inequalities in this association.

Design Cross-sectional multilevel analysis of survey data. Setting Germany.

Participants 3350 men and 3665 women living in 167 municipalities throughout Germany participating in the 'German Health Interview and Examination Survey for Adults' (DEGS1 2008-2011) as part of the national health monitoring.

Outcome Perceived social support as measured by 0slo-3 Social Support Scale.

Results Prevalence of low perceived social support was $11.4 \%$ in men and $11.1 \%$ in women. Low social support was associated in men and women with sociodemographic characteristics that indicate more disadvantaged living situations. Taking these individual-level characteristics into account, municipal-level deprivation was independently associated with low perceived social support in men (OR for the most deprived quintile: $1.80(95 \% \mathrm{Cl} 1.14$ to 2.84$))$, but not in women (OR 1.22 (95\% Cl 0.78 to 1.90$)$ ).

Conclusion The results of our multilevel analysis suggest that there are gender inequalities in the association of municipal-level deprivation with the prevalence of low perceived social support in Germany independent of individual socioeconomic position. Community health interventions aiming at promotion of social support among residents might profit from a further understanding of the observed gender differences.

\section{INTRODUCTION}

Within the framework of recent research on the relevance of contextual factors for health, Wilkinson's hypothesis ${ }^{1}$ opened up a controversial debate on whether population health in rich developed countries is more affected by relative income than by absolute income. His focus on inequalities as measured by relative income facilitated a dispute about the role and respective implications of social capital as
Strengths and limitations of this study

- The investigated study population is part of a nationally representative database on health of adults in Germany.

- The association between area deprivation and perceived social support was analysed by applying valid and reliable measures such as the 0slo-3 Social Support Scale and the German Index of Multiple Deprivation.

- Individual-level sociodemographic and socioeconomic characteristics were comprehensively taken into account.

- For the first time, gender differences in the association of area deprivation and social support were systematically studied. However, a methodical approach for analysis of gender inequalities which goes beyond stratification by the two categories women and men was not possible due to data availability.

a mediator of the outlined income distribution relationship. ${ }^{12}$ Social capital represents a "community-level variable", which is mostly characterised by features of social organisation, such as civic participation, norms of reciprocity and trust in others. ${ }^{2-5}$ Viewed on an individual level, it is analogously measured by a person's social network. ${ }^{3}$ Social support is regarded as a key function of these corresponding social networks. ${ }^{67}$

As an important salutogenic resource in health research, ${ }^{8}$ social support shows a reliable relationship with lower rates of morbidity and mortality, ${ }^{9}$ which influences physical health outcomes through health behaviour, psychological and physiological pathways. ${ }^{9}{ }^{10}$ It can be operationalised as perceived social support, defined as 'the perceived availability of people whom the individual trusts and who make one feel cared for and valued as a person'. ${ }^{11}$ Although social support is 
primarily a personal issue, it is shaped by social structural imperatives. ${ }^{12}$ Thus, all individual social ties taken together merge into something more than simply an aggregate of social support in terms of social cohesion. Viewed on a societal level, this connection has implications for promoting health of communities. ${ }^{12}$ Considering equality within a community as a substantial aspect of a stronger civic community, ${ }^{13}$ evidence suggests that more egalitarian societies are indeed socially more cohesive, for example, exhibiting more trust and involvement in community life. ${ }^{1}$

Within the scope of the income inequality hypothesis, Wilkinson ${ }^{1}$ pointed out to the burden of low relative income which can either be measured within large areas or between economically segregated small areas. Regarding small areas he further specified that population health is not afflicted by persisting inequalities within a deprived neighbourhood, but rather by living in a neighbourhood that as a whole is deprived in relation to the wider society. Following Wilkinson ${ }^{1}$ and the notion of small-area deprivation leading to lowered social capital in a community, it is conceivable that as a consequence population health in general and the personal perception of social support of individuals living in an unequal society are affected.

The contextual and individual determinants of perceived social support such as area deprivation, income, employment, partnership and migrant background have not been investigated thoroughly for men and women, by using a multilevel analytical approach. In this regard, mainly analyses on women have been carried out. ${ }^{14-16}$ None of these studies could reveal an independent association in women. In contrast, to the best of our knowledge, the independent relationship between relative deprivation at a small area level and perceived social support regarding men has not yet been analysed.

Therefore, this cross-sectional study aimed to determine whether relative deprivation at municipality level in Germany is associated with perceived social support, independent of individual socioeconomic position and demographic characteristics. Based on our findings in the literature, we examine gender effects by conducting these analyses separately for men and women.

\section{METHODS}

\section{Study population}

This cross-sectional study is based on the 'German Health Interview and Examination Survey for Adults (DEGS)', which is part of the nationwide health monitoring carried out by the Robert Koch Institute. The first wave (DEGS1 2008-2011) comprises health data for adults aged 18-79 years $(n=7987)$, living in 180 municipalities throughout Germany. Further details about design, methods and nationwide representativity of the DEGS study population have been described elsewhere. ${ }^{17} 18$ The mixed design of DEGS1 allows for both cross-sectional and longitudinal analysis. Longitudinal analysis is based on participants' data derived from the 1998 German National
Health Interview and Examination Survey (GNHIES98). Participants of GNHIES98 were invited to take part in DEGS1 10 years later. Response rate for participants who received only one invitation (DEGS1) was $42 \%(n=4192)$, and for participants with two invitations (GNHIES98 and DEGS1) the response rate was $62 \%(\mathrm{n}=3795)$. To define the study population for this analysis, first, participants of DEGS1 without information about their perceived social support were excluded $(n=121)$. Second, respondents of the GNHIES98 who meanwhile had moved to a different location before taking part in DEGS1 were excluded $(n=410)$. Furthermore, for more accurate estimates the DEGS1 sample was restricted to respondents from municipalities providing data from at least 30 participants (Kreft's $30 / 30$ rule), ${ }^{19}$ which led to an exclusion of 17 municipalities (excluding 441 subjects). Finally, the present analysis was based on a total sample of 3350 men and 3665 women.

\section{Ethics and data protection}

In consideration of information privacy, only analyses based on anonymised individual-level data were conducted. Data used were collected by self-administered questionnaires according to the Federal and State Commissioners for Data Protection guidelines. The implementation of the survey conforms to the principles of the Helsinki Declaration. All participants provided written informed consent prior to survey participation.

\section{Individual-level variables}

Perceived social support

The outcome variable social support was measured by the Oslo-3 Social Support Scale (Oslo-3). ${ }^{20}$ The Oslo-3 is a proxy measure for perceived social support, defined as 'the perceived availability of people whom the individual trusts and who make one feel cared for and valued as a person'. ${ }^{11}$ The scale consists of three questions, asking about the number of close friends the participant has, to which extent other persons are involved in the respondent's life and how the participant perceives the availability of help from neighbours. ${ }^{21}$ Depending on the individually chosen answer categories, a total score can be calculated with values reaching from 3 to 14 (social support: low 3 -8; medium 9-11; high 12-14). In conformity with Buttery et al, ${ }^{22}$ the score was dichotomised into low versus medium/high social support.

\section{Covariates}

With regard to previous studies on sociodemographic determinants of social support, ${ }^{23-25}$ the following sociodemographic characteristics were considered as potential confounders: age (18-64 years/ $\geq 65$ years), education (International Standard Classification of Education (ISCED): high/medium/low), ${ }^{17}$ income (equivalent net household income categorised into tertiles: high/ medium/low), currently employed (yes/no), partnership status (yes/no) and migrant background (yes/no). 


\section{Contextual-level variables}

Area deprivation

The German Index of Multiple Deprivation (GIMD) is an area-based measure of relative deprivation. The GIMD can be applied at the municipal level ${ }^{26}$ and comprises seven differently weighted domains of deprivation: income $(25 \%)$, employment $(25 \%)$, education $(15 \%)$, municipal revenue $(15 \%)$, social capital $(10 \%$; the definition of social capital is based on data on migratory balance of the municipality and participation rates in state parliamentary elections), environment (5\%) and security $(5 \%) .{ }^{27}$ The GIMD applied at present is based on publicly available data of official statistics in Germany with reference year 2010 (GIMD 2010). For the analyses, all 180 DEGS1 municipalities were allocated to quintiles according to their level of deprivation (quintile 1: least deprived/quintile 5: most deprived municipalities).

\section{Categories of political municipalities}

Categories of political municipalities were defined in accordance with the respective number of inhabitants (very small town $<5000$ inhabitants/small town $5000-<20000$ inhabitants/medium sized town $20000-<100000$ inhabitants/large town $\geq 100000$ ).

\section{Statistical methods}

Sociodemographic characteristics at the individual level relevant for social support were first described for men and women separately. Prevalence of low perceived social support was calculated stratified by gender and covariates.

Prior to multilevel modelling, bivariate associations were assessed by logistic regression for men and women separately. To take account of the hierarchical data structure (level 1: individuals; level 2: municipalities) and area-level variances, multilevel logistic regression analyses with municipalities as random intercepts were performed calculating ORs with their 95\% CIs. According to the recommendation of Hosmer et $a l,{ }^{28}$ all independent variables in bivariate logistic regression which were associated with low social support (Wald's $\mathrm{p}<0.2$ ) were included into multivariate analyses.

Four progressively adjusted multilevel models were carried out: model 0 with no covariates; model 1 including only sociodemographic characteristics at the individual level; model 2 additionally analysing municipal deprivation as contextual variable. To take heterogeneity of municipalities in Germany into account, a sensitivity analysis was performed by further adjusting model 2 for categories of political municipalities in accordance with the respective number of inhabitants (model 3). Multicollinearity between independent variables was measured by the variance inflation factor (VIF). VIF values higher than 10 are seen as critical. ${ }^{29}$ The VIF in the second and third models never exceeded values of 3.0.

Referring to the limited evidence given by previous studies, potential effect modification by gender was tested. In accordance with Fleiss $e t a l^{30}$ the $\mathrm{p}$ value of 0.08 as observed for the interaction term was considered relevant.
Therefore, all analyses were conducted separately for men and women. Cross-level interactions between arealevel deprivation and individual-level sociodemographic factors were tested in the second model. Area-level variances (VA) and their SEs are reported for every model and for reasons of better interpretability converted into median ORs (MORs) by applying the formula of Merlo et $a l .{ }^{31}(\mathrm{MOR}=\exp (\sqrt{ }(2 \times \mathrm{VA}) \times 0.6745))$.

The multilevel analyses were performed as complete case analyses which led to an exclusion of 122 (3.64\%) men and $116(3.17 \%)$ women. All statistical analyses were conducted using SAS software V.9.4, estimating the logistic multilevel models with the GLIMMIX procedure. Maximum-likelihood estimations were based on the Laplace method, and the Akaike information criterion (AIC) —as a measure of relative goodness of fit $^{32}$ — was calculated for comparison between the four multilevel models for men and women separately.

In context of health monitoring, survey-specific weighting factors for DEGS1 are available that take sampling and dropout probabilities as well as deviations between the design-weighted net sample and German population statistics 2010 into account. ${ }^{17}$ To allow for more structural interpretation of the parameter estimates, Winship and Radbill ${ }^{33}$ advised to calculate ORs from unweighted analyses, because they are more efficient and have correct standard errors. Therefore, since our analysis was aligned to focus more on the actual association between area deprivation and perceived social support, no population weights were applied.

\section{RESULTS}

The male study population $(n=3350)$ comprises $11.4 \%$ persons with low perceived social support and the female study population $(n=3665) 11.1 \%$. Table 1 shows the distribution of sociodemographic and arealevel characteristics by low social support for women and men separately. Men in the study sample had more often a higher educational level, were more commonly employed and lived more often with a partner in comparison to women.

Men as well as women with low education, low income, not being employed, living without a partner or having a migration background were more likely to have low social support (table 2 ). In men, living in a large town was bivariately associated with low social support. In women, an age above 64 years was associated with low social support. ORs for low social support monotonously increased with increasing area deprivation in men, but not in women.

Results of the multivariate analyses are given in tables 3 and 4 , which are based on progressively adjusted models. In the first model considering only individual characteristics, lower education, lower income, not living with a partner and having a migration background were independently positively associated with low perceived social support in both genders (results for men: table 3, for women: table 4 ). 
Table 1 Individual sociodemographic characteristics, contextual factors and the prevalence of low social support among men and women

\begin{tabular}{|c|c|c|c|}
\hline Men $(\mathrm{N}=3350)$ & & Women $(\mathrm{N}=3665)$ & \\
\hline $\begin{array}{l}\text { Absolute and relative frequency } \\
\text { of sociodemographic and } \\
\text { contextual factors }\end{array}$ & $\begin{array}{l}\text { Prevalence } \\
\text { of low social } \\
\text { support }\end{array}$ & $\begin{array}{l}\text { Absolute and relative frequency } \\
\text { of sociodemographic and } \\
\text { contextual factors }\end{array}$ & $\begin{array}{l}\text { Prevalence } \\
\text { of low social } \\
\text { support }\end{array}$ \\
\hline$\%$ & $\%$ & $\%$ & $\%$ \\
\hline
\end{tabular}

Sociodemographic individual characteristics

Age (years)

\begin{tabular}{|c|c|c|c|c|c|c|}
\hline $18-64$ & 2449 & 73.1 & 10.9 & 2718 & 74.2 & 9.7 \\
\hline$\geq 65$ & 901 & 26. 9 & 12.9 & 947 & 25.8 & 15.2 \\
\hline \multicolumn{7}{|l|}{ Educational level } \\
\hline High & 1282 & 38.3 & 8.7 & 925 & 25.3 & 8.2 \\
\hline Middle & 1696 & 50.7 & 12.4 & 2115 & 57.8 & 10.8 \\
\hline Low & 367 & 11.0 & 16.9 & 619 & 16.9 & 16.5 \\
\hline \multicolumn{7}{|l|}{ Income level } \\
\hline High & 1115 & 33.3 & 8.5 & 1223 & 33.4 & 7.1 \\
\hline Middle & 1137 & 33.9 & 9.9 & 1234 & 33.7 & 11.0 \\
\hline Low & 1098 & 32.8 & 16.0 & 1208 & 32.9 & 15.3 \\
\hline \multicolumn{7}{|l|}{ Employed } \\
\hline Yes & 2090 & 64.0 & 9.7 & 2054 & 57.3 & 8.2 \\
\hline No & 1177 & 36.0 & 13.8 & 1533 & 42.7 & 14.4 \\
\hline \multicolumn{7}{|l|}{ Partner } \\
\hline Yes & 2269 & 67.9 & 10.0 & 2295 & 62.8 & 9.8 \\
\hline No & 1073 & 32.1 & 14.5 & 1360 & 37.2 & 13.2 \\
\hline \multicolumn{7}{|l|}{ Migration background } \\
\hline No & 2808 & 86.2 & 10.6 & 3069 & 85.8 & 10.2 \\
\hline Yes & 448 & 13.8 & 14.7 & 507 & 14.2 & 14.0 \\
\hline \multicolumn{7}{|l|}{ Contextual factors } \\
\hline \multicolumn{7}{|l|}{ Political municipalities } \\
\hline Very small town & 663 & 19.8 & 9.7 & 668 & 18.2 & 10.5 \\
\hline Small town & 816 & 24.4 & 10.2 & 862 & 23.5 & 10.8 \\
\hline Medium town & 985 & 29.4 & 11.2 & 1104 & 30.1 & 10.2 \\
\hline Large town & 886 & 26.4 & 14.2 & 1031 & 28.2 & 12.8 \\
\hline \multicolumn{7}{|c|}{ Area deprivation (GIMD quintiles) } \\
\hline Q1 & 352 & 10.5 & 8.0 & 402 & 11.0 & 9.5 \\
\hline Q2 & 466 & 13.9 & 8.8 & 468 & 12.8 & 9.6 \\
\hline Q3 & 673 & 20.1 & 10.0 & 698 & 19.0 & 12.2 \\
\hline Q4 & 1046 & 31.2 & 12.4 & 1217 & 33.2 & 10.9 \\
\hline Q5 (highest deprivation) & 813 & 24.3 & 14.4 & 880 & 24.0 & 12.2 \\
\hline
\end{tabular}

Political municipalities: very small town $<5000$ inhabitants, small town $5000-<20000$ inhabitants, medium-sized town $20000-<100000$ inhabitants, large town $\geq 100000$ inhabitants; GIMD, German Index of Multiple Deprivation.

In the fully adjusted second model, all individual-level variables remained significant in both genders, except for the independent positive association between currently not being employed and low perceived social support that remained only in women. Regarding the contextual variables in the second model, an independent association between municipal deprivation and low perceived social support was only observed in men (OR for the most deprived quintile 5: 1.80 (95\% CI 1.14 to 2.84)), with odds of low social support increasing along quintile. In model 3 as part of sensitivity analysis all individual-level variables remained significant in both genders, except for the independent positive association between having a migration background and low perceived social support 
Table 2 Bivariate associations of individual sociodemographic characteristics, contextual factors and the odds of low social support by gender

\begin{tabular}{|c|c|c|}
\hline \multirow[b]{2}{*}{ Variables } & Men $(n=3350)$ & Women $(n=3665)$ \\
\hline & OR (95\% Cl) & OR (95\% Cl) \\
\hline \multicolumn{3}{|l|}{ Age (years) } \\
\hline $18-64$ & 1.00 (reference) & 1.00 (reference) \\
\hline$\geq 65$ & 1.21 (0.96 to 1.52$)$ & 1.67 (1.34 to 2.07$)$ \\
\hline \multicolumn{3}{|c|}{ Educational level } \\
\hline High & 1.00 (reference) & 1.00 (reference) \\
\hline Middle & 1.49 (1.17 to 1.90$)$ & 1.35 (1.03 to 1.77$)$ \\
\hline Low & 2.15 (1.53 to 3.00$)$ & 2.20 (1.61 to 3.02$)$ \\
\hline \multicolumn{3}{|c|}{ Income level } \\
\hline High & 1.00 (reference) & 1.00 (reference) \\
\hline Middle & 1.17 (0.88 to 1.56$)$ & $1.62(1.22$ to 2.14$)$ \\
\hline Low & 2.05 (1.57 to 2.67$)$ & 2.36 (1.81 to 3.09$)$ \\
\hline \multicolumn{3}{|l|}{ Employed } \\
\hline Yes & 1.00 (reference) & 1.00 (reference) \\
\hline No & $1.48(1.19$ to 1.85$)$ & $1.87(1.51$ to 2.31$)$ \\
\hline \multicolumn{3}{|l|}{ Partner } \\
\hline Yes & 1.00 (reference) & 1.00 (reference) \\
\hline No & 1.54 (1.24 to 1.91$)$ & $1.40(1.14$ to 1.73$)$ \\
\hline
\end{tabular}

Migration background

\begin{tabular}{|c|c|c|}
\hline No & 1.00 (reference) & 1.00 (reference) \\
\hline Yes & 1.46 (1.09 to 1.95$)$ & 1.44 (1.09 to 1.90$)$ \\
\hline \multicolumn{3}{|c|}{ Political municipalities } \\
\hline Very small town & 1.00 (reference) & 1.00 (reference) \\
\hline Small town & $1.06(0.75$ to 1.50$)$ & 1.03 (0.74 to 1.43$)$ \\
\hline Medium town & 1.18 (0.85 to 1.63$)$ & $0.97(0.71$ to 1.34$)$ \\
\hline Large town & 1.55 (1.13 to 2.14$)$ & $1.25(0.92$ to 1.71$)$ \\
\hline \multicolumn{3}{|c|}{ Area deprivation (GIMD quintiles) } \\
\hline $\begin{array}{l}\text { Q1 (lowest } \\
\text { deprivation) }\end{array}$ & 1.00 (reference) & 1.00 (reference) \\
\hline Q2 & $1.12(0.68$ to 1.84$)$ & 1.02 (0.65 to 1.60$)$ \\
\hline Q3 & 1.28 (0.81 to 2.03$)$ & 1.33 (0.89 to 2.00$)$ \\
\hline Q4 & 1.64 (1.07 to 2.52$)$ & $1.18(0.80$ to 1.72$)$ \\
\hline $\begin{array}{l}\text { Q5 (highest } \\
\text { deprivation) }\end{array}$ & 1.95 (1.26 to 3.00$)$ & 1.33 (0.90 to 1.96$)$ \\
\hline
\end{tabular}

Unadjusted odds ratios (OR) with 95\% confidence intervals (95\% $\mathrm{Cl})$ are given; political municipalities: very small town $<5000$ inhabitants; small town $5000-<20000$ inhabitants; mediumsized town $20000-<100000$ inhabitants; large town $\geq 100000$ inhabitants; GIMD, German Index of Multiple Deprivation.

that remained only in women. Considering categories of political municipalities in this third model, an independent positive association between living in a large town and low perceived social support was only observed in men. The further adjustment for categories of political municipalities in the third model attenuated the effect estimates, but did not abolish the independent association between municipal deprivation and low perceived social support in men. Area-level variations for the individual probability of low perceived social support exist for both genders, with MOR 1.17 for men and MOR 1.40 for women in the respective multilevel null models (tables 3 and 4). The MOR decreased in the subsequent models for both genders, with no remaining municipal-specific variation in the final models only in men. Moreover, there was no interaction between area deprivation and individual sociodemographic characteristics. Comparing the null model with the first model, the values of the AIC decreased for both genders. In the two subsequent models, the AIC further decreased only in men.

\section{DISCUSSION}

The objective of this study was to determine whether area deprivation at the municipality level is associated with low perceived social support, independent of individual-level socioeconomic position. Since evidence of gender-related inequalities is limited, the analysis was carried out separately for men and women. According to our findings, there are gender differences concerning the impact of deprivation at a small area level on perceived social support. While living in most deprived municipalities showed to be independently associated with a higher prevalence of low social support in men, no comparable relation was found for women.

Despite the existing heterogeneity in the applied measures for perceived social support and area deprivation, our findings regarding the female study population are in line with results reported from three other studies with analyses based on multilevel regression. Albor et $a l^{14}$ found no evidence of associations between neighbourhood status and low emotional support in mothers living in England. With focus on urban neighbourhoods in Chicago, Tendulkar et $a l^{15}$ results point into the same direction. The cross-sectional and longitudinal analyses (7-year follow-up) showed that concentrated neighbourhood disadvantage was not a significant predictor of either the level or change in perceived social support in parental caregivers $\left(95 \%\right.$ mothers). Thompson et $a l^{16}$ report results from analyses based on a control group of a longitudinal breast cancer study (2 years) of women living in a Midwestern metropolitan area in the USA. After adding individual-level covariates, they could not find any supporting evidence for direct neighbourhood effects on perceived social support in controls.

The results of our study suggest that with respect to the relation between area deprivation and perceived social support gender inequalities exist within these defined administrative boundaries, even though overall prevalence of perceived social support was comparable between women and men. Social support is a key function of social networks, therefore, it can be hypothesised that men and women build up different types of networks in the municipality they reside in. As a result, the two genders might be differently embedded in the communal structures of 
Table 3 Multivariate associations of individual sociodemographic characteristics, contextual factors and low social support in men $(n=3228)$

\begin{tabular}{|c|c|c|c|c|}
\hline Variables & Model 0 & Model 1 & Model 2 & Model 3 \\
\hline \multicolumn{5}{|l|}{ Age (years) } \\
\hline $18-64$ & & 1.00 (reference) & 1.00 (reference) & 1.00 (reference) \\
\hline$\geq 65$ & - & 1.22 (0.88 to 1.68$)$ & 1.23 (0.89 to 1.71$)$ & 1.21 (0.87 to 1.68$)$ \\
\hline \multicolumn{5}{|l|}{ Educational level } \\
\hline High & & 1.00 (reference) & 1.00 (reference) & 1.00 (reference) \\
\hline Middle & - & 1.32 (1.01 to 1.72$)$ & 1.34 (1.03 to 1.75$)$ & 1.37 (1.05 to 1.79$)$ \\
\hline Low & - & 1.43 (0.97 to 2.11$)$ & 1.50 (1.02 to 2.22$)$ & 1.52 (1.03 to 2.26$)$ \\
\hline \multicolumn{5}{|l|}{ Income level } \\
\hline High & & 1.00 (reference) & 1.00 (reference) & 1.00 (reference) \\
\hline Middle & - & 0.99 (0.73 to 1.34$)$ & 0.93 (0.69 to 1.27$)$ & 0.96 (0.71 to 1.30$)$ \\
\hline Low & - & 1.56 (1.16 to 2.10$)$ & $1.42(1.05$ to 1.92$)$ & 1.49 (1.10 to 2.01$)$ \\
\hline \multicolumn{5}{|l|}{ Employed } \\
\hline Yes & & 1.00 (reference) & 1.00 (reference) & 1.00 (reference) \\
\hline No & - & 1.31 (0.98 to 1.74$)$ & 1.29 (0.96 to 1.72$)$ & 1.28 (0.96 to 1.70$)$ \\
\hline \multicolumn{5}{|l|}{ Partner } \\
\hline Yes & & 1.00 (reference) & 1.00 (reference) & 1.00 (reference) \\
\hline No & - & 1.54 (1.21 to 1.96$)$ & 1.53 (1.20 to 1.95$)$ & 1.50 (1.18 to 1.91$)$ \\
\hline \multicolumn{5}{|l|}{ Migration background } \\
\hline No & & 1.00 (reference) & 1.00 (reference) & 1.00 (reference) \\
\hline Yes & - & 1.39 (1.03 to 1.88$)$ & 1.44 (1.07 to 1.95$)$ & 1.33 (0.98 to 1.81$)$ \\
\hline \multicolumn{5}{|l|}{ Political municipalities } \\
\hline Very small town & - & - & - & 1.00 (reference) \\
\hline Small town & - & - & - & $1.06(0.74$ to 1.51$)$ \\
\hline Medium town & - & - & - & 1.24 (0.88 to 1.75$)$ \\
\hline Large town & - & - & - & 1.53 (1.08 to 2.16$)$ \\
\hline \multicolumn{5}{|c|}{ Area deprivation (GIMD quintiles) } \\
\hline Q1 (lowest deprivation) & - & - & 1.00 (reference) & 1.00 (reference) \\
\hline Q2 & - & - & 1.14 (0.68 to 1.91$)$ & 1.10 (0.65 to 1.86$)$ \\
\hline Q3 & - & - & 1.19 (0.74 to 1.94$)$ & 1.08 (0.66 to 1.76$)$ \\
\hline Q4 & - & - & 1.56 (1.00 to 2.44$)$ & 1.34 (0.84 to 2.13$)$ \\
\hline Q5 (highest deprivation) & - & - & 1.80 (1.14 to 2.84$)$ & 1.66 (1.04 to 2.63$)$ \\
\hline \multicolumn{5}{|l|}{ Variances } \\
\hline $\mathrm{V}_{\mathrm{A}}(\mathrm{SE})$ & $0.01804(0.06021)$ & $0.01372(0.05995)$ & 0 & 0 \\
\hline MOR & 1.17 & 1.15 & 1.00 & 1.00 \\
\hline \multicolumn{5}{|l|}{ Fit statistic } \\
\hline AIC & 2269.79 & 2224.36 & 2218.37 & 2216.94 \\
\hline
\end{tabular}

Adjusted odds ratios (OR) with 95\% confidence intervals (95\% Cl) are given.

AIC, akaike information criterion; GIMD, German Index of Multiple Deprivation; MOR, median odds ratio; SE, standard error; VA, area level variances.

their municipality and thus conceivably be different in the way their perception of social support is affected by the deprivation status of their municipality. On a societal level, gender differences concerning social networks have already been described regarding participation in civic associations. While men predominate in community associations, political parties and labour unions, women by contrast are more associated with organisations that are stronger related to traditional female roles. ${ }^{34}$ These are associations concerned with education, arts, religion, social welfare services and women's groups. ${ }^{54} 35$ Referring to results of their multivariate analysis, Norris and 
Table 4 Multivariate associations of individual sociodemographic characteristics, contextual factors and low social support in women $(n=3549)$

\begin{tabular}{|c|c|c|c|c|}
\hline Variables & Model 0 & Model 1 & Model 2 & Model 3 \\
\hline \multicolumn{5}{|l|}{ Age (years) } \\
\hline $18-64$ & & 1.00 (reference) & 1.00 (reference) & 1.00 (reference) \\
\hline$\geq 65$ & - & $1.12(0.84$ to 1.45$)$ & $1.12(0.85$ to 1.50$)$ & 1.12 (0.84 to 1.45$)$ \\
\hline \multicolumn{5}{|l|}{ Educational level } \\
\hline High & & 1.00 (reference) & 1.00 (reference) & 1.00 (reference) \\
\hline Middle & - & 1.15 (0.86 to 1.54$)$ & $1.15(0.86$ to 1.54$)$ & 1.16 (0.87 to 1.55$)$ \\
\hline Low & - & 1.46 (1.02 to 2.08$)$ & 1.47 (1.03 to 2.11$)$ & 1.49 (1.04 to 2.13 ) \\
\hline \multicolumn{5}{|l|}{ Income level } \\
\hline High & & 1.00 (reference) & 1.00 (reference) & 1.00 (reference) \\
\hline Middle & - & 1.40 (1.04 to 1.87$)$ & $1.38(1.03$ to 1.85$)$ & 1.40 (1.04 to 1.88$)$ \\
\hline Low & - & 1.76 (1.31 to 2.37$)$ & 1.74 (1.29 to 2.35$)$ & 1.78 (1.31 to 2.41$)$ \\
\hline \multicolumn{5}{|l|}{ Employed } \\
\hline Yes & & 1.00 (reference) & 1.00 (reference) & 1.00 (reference) \\
\hline No & - & 1.62 (1.24 to 2.12$)$ & 1.61 (1.23 to 2.10$)$ & 1.59 (1.22 to 2.08 ) \\
\hline \multicolumn{5}{|l|}{ Partner } \\
\hline Yes & & 1.00 (reference) & 1.00 (reference) & 1.00 (reference) \\
\hline No & - & 1.32 (1.06 to 1.66$)$ & $1.33(1.06$ to 1.90$)$ & 1.30 (1.04 to 1.63 ) \\
\hline \multicolumn{5}{|l|}{ Migration background } \\
\hline No & & 1.00 (reference) & 1.00 (reference) & 1.00 (reference) \\
\hline Yes & - & 1.41 (1.06 to 1.89$)$ & $1.42(1.06$ to 1.90$)$ & 1.38 (1.03 to 1.85$)$ \\
\hline \multicolumn{5}{|l|}{ Political municipalities } \\
\hline Very small town & - & - & - & 1.00 (reference) \\
\hline Small town & - & - & - & 1.01 (0.70 to 1.45$)$ \\
\hline Medium town & - & - & - & 1.04 (0.73 to 1.48$)$ \\
\hline Large town & - & - & - & 1.23 (0.86 to 1.77$)$ \\
\hline \multicolumn{5}{|c|}{ Area deprivation (GIMD quintiles) } \\
\hline Q1 (lowest deprivation) & - & - & 1.00 (reference) & 1.00 (reference) \\
\hline Q2 & - & - & 1.02 (0.62 to 1.70$)$ & 0.99 (0.60 to 1.64$)$ \\
\hline Q3 & - & - & 1.31 (0.84 to 2.04$)$ & 1.23 (0.79 to 1.94$)$ \\
\hline Q4 & - & - & $1.10(0.72$ to 1.67$)$ & 1.00 (0.64 to 1.55$)$ \\
\hline Q5 (highest deprivation) & - & - & $1.22(0.78$ to 1.90$)$ & 1.14 (0.73 to 1.79$)$ \\
\hline \multicolumn{5}{|l|}{ Variances } \\
\hline$V_{A}(S E)$ & $0.08553(0.06283)$ & $0.06047(0.06129)$ & $0.054290(0.06079)$ & $0.04690(0.06004)$ \\
\hline MOR & 1.40 & 1.33 & 1.31 & 1.29 \\
\hline \multicolumn{5}{|l|}{ Fit statistic } \\
\hline AIC & 2396.17 & 2336.90 & 2342.43 & 2346.39 \\
\hline
\end{tabular}

Adjusted odds ratios (OR) with 95\% confidence intervals $(95 \% \mathrm{Cl})$ are given.

AIC, akaike information criterion; GIMD, German Index of Multiple Deprivation; MOR, median odds ratio; SE, standard error; VA, area level variances.

Inglehart ${ }^{34}$ suggest that the gender gap in formal associational membership could be a reflection of the way in which women and men differ in their informal social networks. While spending time informally with workmates and friends was positively correlated with participation in formal associations, spending time with family and immediate relatives-which appears to be more common among women ${ }^{34}{ }^{36}$ - is less likely to drag people into joining formal organisations and community groups. ${ }^{34}$

The GIMD measure for municipal-level deprivation was derived from official statistics. Since social capital deduced from data about migratory balance of the 
municipality together with participation in state parliamentary elections is one of the GIMD domains weighted with $10 \%$, it is important to point out that an exhaustive conceptual distinction between this domain and perceived social support at the individual level cannot be made. Nonetheless, the GIMD comprises mainly labour market issues (inhabitants' income, employment, municipal revenues). Since men participate more in community associations, political parties and labour unions and their informal networks in general are more related to workmates and friends compared with women, it can be assumed that they are also stronger embedded in the formal structures of the municipality they live in. Regarding social support as a key function of social networks-with men in general being more associated within formal networks-it can further be assumed that a higher involvement in formal networks might lead to higher vulnerability towards the mentioned deprivation-related aspects of the political municipality individuals reside in. As a consequence, living in the most deprived municipalities could increase the chance for low perceived social support.

\section{Strengths and limitations}

Considering non-response bias in connection with the social isolation hypothesis, ${ }^{37}$ a limitation of this study is that it is likely that individuals with low perceived social support could have participated less in the survey. Furthermore, as a consequence of increasing area deprivation being associated with higher non-participation, deprivation level of residential area might as well predict non-response ${ }^{38}$ However, we assume that non-response could result in an underestimation of the investigated association. The fact that the response rate of the DEGS1 survey was below $70 \%$ and that unweighted statistical analyses were performed might be interpreted as another limitation. It has been discussed before that a high response rate does not necessarily imply unbiased response. ${ }^{18}$ In addition, Kamtsiuris $e t a l^{18}$ stated that the DEGS1 study population has a high representativity as ascertained through comparing responder and non-responder in terms of sociodemographic and health-related characteristics.

Using data of the cross-sectional survey DEGS1, we analysed gender inequalities based on the two categories women and men. Although already taking individual socioeconomic position into account, relying on this male/female binary may mask variations inherent to populations. ${ }^{36}$ Unfortunately, with the data at hand we were not able to further explore gender as a complex relational concept that depicts processes within a society, considering changes according to time and place. ${ }^{39} 40$ In addition, we focused our analysis on the association between area deprivation and social support, thereby controlling for numerous sociodemographic covariates. Nonetheless, the lower perceived social support might still be confounded by other variables such as poorer mental health. A further limitation is that estimates from cross-sectional analyses do not allow for causal interpretations.
Strengths of this study are the applied measures for perceived social support and multiple area deprivation. The use of the Oslo-3 has proven to be adequate in measuring perceived social support and is therefore recommended in European health monitoring. ${ }^{20}{ }^{21}$ The GIMD has shown its ability to effectively assess area deprivation at municipality level in Germany. ${ }^{24}{ }^{25} \mathrm{~A}$ further strength is the DEGS1 study population that is part of a nationally representative database on health of adults in Germany and based on a two-stage stratified random sampling from local population registries. ${ }^{17}$

In health research, a sound conception of place itself as well as explanatory models for observed empirical relations between place and health are scarce. ${ }^{41}{ }^{42}$ Operationalisation of the small-area context in health research has been assumed to be often opportunistic and based on data availability. ${ }^{42}$ Regarding municipalities as a small-area context in the analysis at hand, the geographical boundaries can be clearly defined. Beyond that, as a political subdivision of the state, each municipality has its own local government accountable for its respective inhabitants.

\section{CONCLUSION}

The results of our multilevel analysis may suggest that in Germany higher municipal-level deprivation is associated with a higher prevalence of low perceived social support in men, independent of individual sociodemographic characteristics. Further research is required to understand the mechanisms underlying the observed gender inequalities.

Contributors EM was primarily responsible for study concept and data analysis. She drafted and wrote the manuscript. LEK assisted in the conception and design of the work for the data provider and revised an earlier draft. WM provided data of the German Index of Multiple Deprivation, advised on its use and gave critical feedback on the manuscript draft. GB contributed to study concept, data analysis and manuscript preparation. All authors approved the final manuscript.

Funding This research received no specific grant from any funding agency in the public, commercial or not-for-profit sectors.

Competing interests None declared.

Patient consent Obtained.

Ethics approval DEGS1 was approved by the local ethics committee at CharitéUniversitätsmedizin Berlin in October 2008 (ethics approval application document number: EA2/047/08).

Provenance and peer review Not commissioned; externally peer reviewed.

Data sharing statement Datasets of the DEGS1 study are available for scientific use from fdz@rki.de

Open Access This is an Open Access article distributed in accordance with the Creative Commons Attribution Non Commercial (CC BY-NC 4.0) license, which permits others to distribute, remix, adapt, build upon this work non-commercially, and license their derivative works on different terms, provided the original work is properly cited and the use is non-commercial. See: http://creativecommons.org/ licenses/by-nc/4.0/

(c) Article author(s) (or their employer(s) unless otherwise stated in the text of the article) 2018. All rights reserved. No commercial use is permitted unless otherwise expressly granted.

\section{REFERENCES}

1. Wilkinson RG. Inequality and the social environment: a reply to Lynch et al. J Epidemiol Community Health 2000;54:411-3. 
2. Lynch J, Due P, Muntaner C, et al. Social capital--is it a good investment strategy for public health? J Epidemiol Community Health 2000;54:404-8.

3. Kawachi I, Kennedy BP, Lochner K, et al. Social capital, income inequality, and mortality. Am J Public Health 1997;87:1491-8.

4. Kawachi I, Berkman LF. Social capital, social cohesion, and health. In: Berkman LF, Kawachi I, Glymour MM, eds. Social epidemiology. Oxford: Oxford University Press, 2014:290-319.

5. Putnam RD. Bowling alone: the collapse and revival of American community. New York: Simon \& Schuster, 2000.

6. Berkman LF, Krishna A. Social network epidemiology. In: Berkman LF, Kawachi I, Glymour MM, eds. Social epidemiology. Oxford: Oxford University Press, 2014:234-89.

7. Schwarzer KAA, Weinman J. Health psychology: Wiley, 2004.

8. Antonovsky A. Health, stress, and coping: Jossey-Bass, 1979.

9. Uchino BN. Social support and health: a review of physiological processes potentially underlying links to disease outcomes. J Behav Med 2006;29:377-87.

10. Berkman LF, Glass T. Social integration, social networks, social support, and health. In: Berkman LF, Kawachi I, eds. Social epidemiology. New York: Oxford University Press, 2000:137-73.

11. Korkeila J, Tuomi-Nikula A, Wahlbeck K, et al. Proposal for a harmonised set of mental health indicators. In: Lavikainen JF, Lehtinen TV, eds. Improving mental health information in europe proposal of the MINDFUL project. European Union: Stakes:112.

12. Stansfeld S. Social cohesion and social support. In: Marmot M, Wilkinson RG, eds. Social determinants of health. Oxford: Oxford University Press, 2008:148-71.

13. Putnam RD, Leonardi R, Nanetti RY. Making democracy work: civic traditions in modern Italy: Princeton University Press, 1993.

14. Albor C, Uphoff EP, Stafford M, et al. The effects of socioeconomic incongruity in the neighbourhood on social support, self-esteem and mental health in England. Soc Sci Med 2014;111:1-9.

15. Tendulkar SA, Koenen KC, Dunn EC, et al. Neighborhood influences on perceived social support among parents: findings from the project on human development in Chicago neighborhoods. PLoS One 2012;7:e34235.

16. Thompson T, Rodebaugh TL, Pérez M, et al. Influence of neighborhood-level factors on social support in early-stage breast cancer patients and controls. Soc Sci Med 2016;156:55-63.

17. Scheidt-Nave C, Kamtsiuris P, Gößwald A, et al. German health interview and examination survey for adults (DEGS) - design, objectives and implementation of the first data collection wave. BMC Public Health 2012;12:730.

18. Kamtsiuris $P$, Lange $M$, Hoffmann R, et al. [The first wave of the German Health Interview and Examination Survey for Adults (DEGS1): sample design, response, weighting and representativeness]. Bundesgesundheitsblatt Gesundheitsforschung Gesundheitsschutz 2013;56:620-30.

19. Hox JJ. Multilevel analysis: techniques and applications. New Jersey, USA: Lawrence Erlbaum Associates, 2002.

20. Meltzer $\mathrm{H}$. Development of a common instrument for mental health. In: Nosikov A, Gudex C, eds. Developing common instruments for health surveys. Amsterdam: IOS Press, 2003.

21. Kilpeläinen K, Aromaa A, ECHIM Core Group, eds. European health indicators: development and initial implemantation. Final report of the ECHIM project. Helsinki: National Public Health Institute, 2008.

22. Buttery AK, Busch MA, Gaertner B, et al. Prevalence and correlates of frailty among older adults: findings from the German health interview and examination survey. BMC Geriatr 2015;15:22.
23. Dalgard OS. Social inequalities in mental health in Norway: possible explanatory factors. Int J Equity Health 2008;7:27.

24. Dalgard OS, Mykletun A, Rognerud M, et al. Education, sense of mastery and mental health: results from a nation wide health monitoring study in Norway. BMC Psychiatry 2007;7:20.

25. Dalgard OS, Thapa SB, Hauff E, et al. Immigration, lack of control and psychological distress: findings from the Oslo Health Study. Scand J Psychol 2006;47:551-8.

26. Maier W, Holle R, Hunger M, et al. The impact of regional deprivation and individual socio-economic status on the prevalence of Type 2 diabetes in Germany. A pooled analysis of five population-based studies. Diabet Med 2013;30:e78-e86.

27. Maier W, Fairburn J, Mielck A. [Regional deprivation and mortality in Bavaria. Development of a community-based index of multiple deprivation]. Gesundheitswesen 2012;74:416-25.

28. Hosmer JDW, Lemeshow S, Sturdivant RX. The multiple logistic regression model: Applied Logistic Regression: John Wiley \& Sons, Inc, 2013:35-7.

29. Katz MH. Multivariable analysis: a practical guide for clinicians and public health researchers. Cambridge: Cambridge University Press, 2011.

30. Fleiss JL, Levin B, Paik MC. Statistical methods for rates and proportions: Wiley, 2013.

31. Merlo J, Chaix B, Ohlsson $\mathrm{H}$, et al. A brief conceptual tutorial of multilevel analysis in social epidemiology: using measures of clustering in multilevel logistic regression to investigate contextual phenomena. J Epidemiol Community Health 2006;60:290-7.

32. Akaike H. A new look at the statistical model identification. IEEE Trans Automat Contr 1974;19:716-23.

33. Winship C, Radbill L. Sampling weights and regression analysis. Sociol Methods Res 1994;23:230-57.

34. Norris P, Inglehart R. Gendering social capital. Gender and social capital, 2013:73-98.

35. Bundesministerium für Familie $\mathrm{S}$, Frauen und Jugend (BMFSFJ). Hauptbericht des Freiwilligensurveys 2009. Zivilgesellschaft, soziales Kapital und freiwilliges Engagement in Deutschland 1999 - 2004 2009. Berlin: Bundesministerium für Familie, Senioren, Frauen und Jugend, 2010.

36. Moore G. Structural determinants of men's and women's personal networks. Am Sociol Rev 1990;55:726-35.

37. Groves RM, Couper MP. Nonresponse in household interview surveys. New York, NY: Wiley, 1998.

38. Goodman A, Gatward R. Who are we missing? Area deprivation and survey participation. Eur J Epidemiol 2008;23:379-87.

39. Johnson JL, Repta R. Sex and gender. Beyond the binaries. In: Oliffe JL, Greaves L, eds. Designing and conducting gender, sex, and health research. Los Angeles/London/New Dehli/Singapore/ Washington DC: SAGE Publications, 2012:17-37.

40. Annandale E, Hunt K. Gender inequalities in health: research at the crossroads. In: Annandale E, Hunt K, eds. Gender inequalities in health. Buckingham/Philadelphia: Open University Press, 2000:1-35.

41. Bernard P, Charafeddine R, Frohlich KL, et al. Health inequalities and place: a theoretical conception of neighbourhood. Soc Sci Med 2007;65:1839-52.

42. Voigtländer S, Vogt V, Mielck A, et al. Explanatory models concerning the effects of small-area characteristics on individual health. Int $J$ Public Health 2014;59:427-38. 